ESJ Social Sciences

\title{
Multiplicateurs budgétaires et règles de la politique budgétaire au Maroc : Une étude en SVAR
}

\author{
Jaouad Laamire \\ Omar Zirari
}

Laboratoire d'Economie Appliquée, Faculté des Sciences Juridiques, Economiques et Sociales d'Agdal, UM5 de Rabat, Maroc

Doi:10.19044/esj.2021.v17n5p146

Submitted: 21 August 2020

Accepted: 20 January 2021

Published: 28 February 2021
Copyright 2021 Author(s)

Under Creative Commons BY-NC-ND

4.0 OPEN ACCESS

Cite As:

Laamire J. \& Zirari O. (2021). Multiplicateurs budgétaires et règles de la politique budgétaire au Maroc : Une étude en SVAR. European Scientific Journal, ESJ, 17(5), 146.

https://doi.org/10.19044/esj.2021.v17n5p146

\section{Résumé}

Cet article a pour objectif d'évaluer l'effet multiplicateur dans le cadre d'une règle de politique budgétaire. Plus précisément, c'est une étude de trois modèles VAR Structurelles (S-VAR), avec des données étalées de 1970 jusqu'à 2019 pour le cas du Maroc, concernant les réactions des multiplicateurs budgétaires suite aux différents chocs sur les dépenses publiques d'investissement, de consommations publiques (ordinaire) et dépenses totales. Trois résultats peuvent être mis en évidence. Premièrement, les effets multiplicateurs d'un choc positif des trois variables budgétaires sur le produit intérieur brut réel (PIB) sont tous positifs à la période du choc. Deuxièmement, une augmentation des dépenses d'investissement de $1 \%$ s'accompagne d'une augmentation de $0.28 \%$ du PIB réel Marocain. Troisièmement, les chocs positifs sur les dépenses ordinaires et sur les dépenses totales ont des effets contemporains positifs de 0.43 et 0.56 respectivement. Finalement, le constat le plus important est le fait que les effets contemporains des chocs de dépenses ordinaires sont plus importants que ceux des dépenses d'investissement et des dépenses totales.

Mots clés : Politique budgétaire ; règle budgétaire ; multiplicateur budgétaire, SVAR 


\title{
Fiscal multipliers and fiscal policy rules in Morocco: A Structural VAR Approach
}

\author{
Jaouad Laamire \\ Omar Zirari \\ Laboratory of Applied Economics, Faculty of Law, Economics and Social \\ Sciences of Agdal, Mohammed V University of Rabat, Morocco
}

\begin{abstract}
This paper aims to evaluate the multiplier effect in the context of a fiscal policy rule. More specifically, it is a study of three S-VAR models, with data from 1970 to 2019 for the case of Morocco, concerning the reactions of fiscal multipliers to different shocks on public investment expenditure, public consumption (ordinary) and total expenditure. Three results can be highlighted. First, the multiplier effects of a positive shock of the three fiscal variables on real GDP are all positive during the shock period. Second, a 1 percent increase in investment spending is accompanied by a 0.28 percent increase in Morocco's real GDP. Third, positive shocks to current and total spending have positive contemporaneous effects of 0.43 and 0.56, respectively. Finally, the most important finding is that the contemporaneous effects of shocks to ordinary spending are larger than those to capital and total spending.
\end{abstract}

Keywords: Fiscal policy; fiscal rule; fiscal multiplier, SVAR

\section{Introduction}

Le cadre budgétaire doit servir deux finalités : promouvoir la discipline dans les finances publiques et permettre la conduite de politiques de stabilisation; ces normes sont actuellement appliquées dans un grand nombre de pays, aussi bien développés qu'en développement. De plus, dans le choix d'un objectif spécifique pour une règle budgétaire, il est nécessaire de trouver un compromis entre l'efficience économique et des considérations plus pratiques. Afin d'opérationnaliser une règle budgétaire, il faut également définir un horizon pertinent dans le but d'atténuer le dilemme crédibilité-flexibilité des règles budgétaires tout en améliorant la transparence budgétaire.

En gros, les règles budgétaires ont pour objectif d'éviter les déséquilibres structurels persistants des finances publiques, de limiter le caractère pro-cyclique des politiques publiques et de maintenir les ratios de la dette publique à des niveaux soutenables. Plus fondamentalement, elles 
ont pour but de corriger le biais inhérent en faveur des déficits publics associés aux distorsions d'incitations auxquelles les décideurs font face.

D'une part, les débats théoriques apportent comme idée qu'un déficit budgétaire important risque d'affecter l'activité économique, chose illustrée dans la période critique qui a caractérisé les finances publiques européennes ces dernières années, ce qui nécessite la mise en place d'une règle budgétaire précise et efficiente. D'autre part et au-delà des discussions théoriques et institutionnelles, les débats sur cette question pour un pays émergent comme le Maroc comportent une autre dimension. En effet, quelle sera la règle budgétaire efficace pour le cas du Maroc ?

Dans ce cadre, le présent travail a pour objet d'étudier l'impact des règles budgétaires sur l'activité économique au Maroc à travers le mécanisme du multiplicateur budgétaire. Plus précisément, l'effet multiplicateur résultant d'une variation au niveau des dépenses publiques sur l'activité économique. Pour ce faire, la première section reviendra brièvement sur les débats théoriques autour de ce sujet. Dans une deuxième section, les différentes approches empiriques développées pour identifier les règles budgétaires. Une dernière section passera en revue la méthodologie, les données et les résultats du modèle SVAR utilisé pour cet objet.

\section{Politique budgétaire des règles budgétaires}

\subsection{Cadrage macroéconomique de la politique budgétaire}

La politique macroéconomique a vu le jour avec J. M. Keynes (18831946) et sa célèbre " théorie générale de l'emploi, de la monnaie et de l'intérêt » (1936), au sens d'une action des pouvoirs publics sur des variables macroéconomiques considérées comme instruments dans le but de faire varier le niveau général des prix, le taux de croissance économique ou le niveau de l'emploi.

Le principe de la demande «effective » a constitué le premier fondement théorique de la politique économique conjoncturelle, il explique que le niveau d'emploi est déterminé par le niveau de la demande globale anticipée, appelée par Keynes «effective demand».

Puis John Hicks (1936) a effectué une relecture en donnant naissance à son célèbre modèle IS-LM qui est un cadre macroéconomique privilégié de l'analyse de la politique économique d'où Hicks annonce que l'analyse économique gagnerait à désagréger le revenu global pour tenir compte de sa distribution. Ce modèle a été par la suite augmenté d'une courbe d'équilibre de la balance des paiements (BP) pour rendre compte de l'ouverture croissante des économies et pour séparer l'analyse de l'équilibre général en régime de change fixe de son analyse en régime de change flottant, ce modèle IS-LM-BP a tendance à montrer que, dans la nouvelle situation caractérisée par la libéralisation des échanges extérieurs, la politique 
budgétaire perdait de son efficacité, contrairement à l'enseignement central de la théorie générale de Keynes. Le deuxième enseignement tiré de ce modèle Mundell-Fleming est le fameux triangle d'incompatibilité selon lequel une économie nationale ouverte ne peut pas atteindre simultanément les trois objectifs suivants : avoir un régime de change fixe ; disposer d'une politique monétaire autonome; et avoir une parfaite libre circulation des capitaux. Ensuite, ce modèle a été augmenté de la courbe de Phillips pour intégrer l'emploi et les prix absents au départ de l'analyse néo-keynésienne.

La contre-révolution monétariste des années 1960 conduit à l'abandon du modèle IS-LM pour un modèle d'équilibre général, afin de faire la part belle aux idées des nouveaux classiques et intégrer les idées des néokeynésiens.

Toutefois, à partir des années 1980, il était devenu de plus en plus difficile de distinguer la macroéconomie théorique des scientifiques de la macroéconométrie empirique des ingénieurs. Cette fusion s'est accentuée avec le développement du modèle DGSE (dynamic general stochastic equilibrium) qui constitue depuis les années 1990 une nouvelle synthèse des nouveaux classiques et des nouveaux keynésiens.

\subsection{Politique budgétaire et règles budgétaires}

À partir de la récession du début des années 1990, les déséquilibres budgétaires récurrents des pays de l'OCDE sont devenus des objets de préoccupation majeure, les velléités de reprise de contrôle des finances publiques maintes fois proclamées n'ayant pas produit de résultats significatifs, certains pays ont commencé à se donner des règles formelles plus strictes. En 2009, quelque 80 pays avaient mis en place un cadre contraignant leur politique budgétaire, de leur propre chef ou pour respecter des engagements internationaux. Alors, le débat sur les règles budgétaires n'est pas un phénomène récent, la plus ancienne règle budgétaire est la règle d'équilibre budgétaire simple.

Cependant, la Grande dépression ${ }^{1}$ a souligné le caractère intenable de cette règle pendant les périodes de récession sévère, ainsi, les règles ont cédé la place à la politique budgétaire discrétionnaire qui a été développé après la Seconde Guerre mondiale.

En ce moment, le débat sur les règles budgétaires pourrait traite essentiellement des questions telles que l'incohérence temporelle dans le comportement des gouvernements, le biais du déficit des gouvernements, et le cycle des affaires politiques (Alesina et Perotti, 1994; Corsetti et Roubini, 1996; Drazen, 2004; Kydland et Prescott, 1977).

${ }^{1}$ La Grande Dépression, dite également «crise économique des années 1930 », est la période de l'histoire mondiale allant de la crise de surproduction de 1929 aux États-Unis jusqu'à la Seconde Guerre mondiale. 
La crise des années 2007-2012 est l'une des raisons principales qui ont contribué à l'instauration des règles budgétaires, qui seraient nécessaires, car les gouvernements ne sont pas bienveillants.

\section{Multiplicateur budgétaire et règles budgétaires.}

L'examen de la littérature empirique consacrée à l'indentification des effets des chocs budgétaires sur l'activité économique révèle l'émergence de plusieurs approches, ce travail s'intéresse uniquement aux règles budgétaires relatives aux dépenses qui imposent dans la plupart des cas une limite stricte et permanente sur les dépenses totales, les dépenses primaires, ou les dépenses courantes.

\subsection{Chocs des dépenses publiques}

Mises à part les autres composantes de la politique budgétaire, les dépenses publiques sont devenues un outil de plus en plus important dans la lutte contre la crise financière dans les pays à économie développée (Mathieu et Sterdyniak, 2009). Il est admis que les dépenses publiques d'investissement ont un impact particulièrement important sur la croissance économique canadienne à long terme, notamment à travers une amélioration de la productivité, l'augmentation de la rentabilité, la création d'emplois additionnels, la compétitivité des marchés, etc.

Il existe plusieurs études antérieures qui analysent ceci, ainsi, depuis plusieurs décennies, investiguer les effets de la politique budgétaire sur la croissance économique de certains pays a été l'objet de plusieurs travaux de recherche.

Barro (1981), en utilisant une modèle dynamique stochastique, a estimé les effets sur la production globale des changements temporaires et permanents dans les dépenses publiques aux États-Unis, à travers ses analyses basées principalement sur les théories macroéconomiques et des modèles empiriques, il conclut que les changements temporaires dans les dépenses publiques des États- Unis se distinguent des changements permanents en raison de leurs effets positifs différenciés respectivement sur la production et le taux d'intérêt réel, il ajoute que ces effets expansionnistes significatifs sont plus élevés sur le PIB réel lorsque les changements dans les dépenses publiques sont temporaires plutôt que permanents, tandis que l'effet positif sur le taux d'intérêt réel après taxes est prédit seulement par les changements temporaires.

Baxter et King (1993) s'inspirant du modèle néoclassique de base, arrivent à un résultat tout à fait différent. Selon ces auteurs, des changements permanents dans les dépenses publiques ont des effets importants sur l'activité macroéconomique quand ces changements sont financés par des taxes forfaitaires, ils avancent qu'il est fort probable que l'effet multiplicateur 
soit plus grand que 1 à long terme, ce résultat est également possible à court terme pourvu que l'élasticité du facteur travail soit très élevée. Aussi, selon ces deux économistes : des changements permanents dans les dépenses publiques engendrent des effets de production plus grands que des changements temporaires dans les dépenses publiques.

Par ailleurs, s'appuyant principalement sur l'approche Vecteur Autoregressif (VAR), plusieurs études ont analysé les effets des composantes publiques budgétaires sur la croissance économique de quelques pays développés.

Blanchard et Perotti (2002), voulant caractériser les effets dynamiques des chocs de dépenses publiques et des taxes sur l'activité économique des États-Unis sur la période après- guerre, trouvent qu'aux chocs positifs de dépenses publiques s'associent toujours un effet positif sur la production (PIB), tandis qu'à des chocs positifs de taxes correspondent un effet négatif. Autrement dit, lorsque les dépenses publiques augmentent, le PIB augmente également ; mais une augmentation dans les taxes entraine une chute de la production. Dans plusieurs cas, les effets multiplicateurs sont petits, souvent proches de 1.

Perotti et al. (2007), en analysant les effets de la politique budgétaire sur l'activité économique (PIB, taux d'inflation et taux d'intérêt à long terme) en Italie, leurs résultats montrent que les chocs de dépenses publiques de biens et services ont un important effet largement significatif et robuste sur l'activité économique : un choc exogène de $1 \%$ augmente le PIB réel privé de $0,6 \%$ après 3 trimestres, la réponse du PIB privé tend vers zéro après 2 ans, reflétant ainsi un décalage de la persistance relativement faible du choc de dépenses publiques. De plus, ils ont conclu que la croissance dans l'activité économique est déterminée à la fois par les réponses positives de la consommation et de l'investissement sur lesquels les effets sont aussi positifs.

Dans d'autres cas d'analyse, les résultats obtenus des problèmes spécifiques étudiés sont interprétés sur la base des modèles d'équilibre général développés par les théories macroéconomiques :

Cogan et al. (2010) utilisent cinq modèles macroéconomiques (NéoKeynésiens DSGE et keynésien traditionnel) structurels d'estimation empirique pour évaluer les effets des programmes de relance fiscale dans la région Européenne afin de stimuler la croissance économique après la période de la crise financière $2007-2008^{2}$. Les résultats ont montré que le comportement symétrique du PIB par rapport à une réduction dans les dépenses publiques est justifié, et les dépenses publiques et les anticipations

${ }^{2}$ La crise financière internationale de 2007-2008 est une crise financière connue par un besoin massif de liquidité et principalement par des problèmes de solvabilité tant au niveau des banques que des États. 
du secteur privé jouent un rôle respectif très important dans la variation de la croissance du PIB. Les auteurs ajoutent que l'impact négatif des dépenses publiques retardées et l'effet de richesse négatif sur la consommation privée des taxes futures hautement anticipées réduisent l'impact positif de la relance fiscale et affectent à la baisse les activités économiques des pays de cette région.

Woodford (2010), dans son étude axée sur l'analyse des déterminants clés de l'effet multiplicateur du PIB des dépenses publiques dans les modèles Néo-Keynésien couramment utilisés dans le contexte d'une politique de stabilisation monétaire, a conclu sur la taille d'un effet multiplicateur d'une augmentation dans les dépenses publiques sur la production agrégée: «l'effet multiplicateur est plus grand que 1 , et bien au-dessus de $1 »$. On souligne que même dans des circonstances de dépression type, il y a un large effet d'une variation dans les dépenses publiques sur la production globale, ainsi qu'une augmentation du bien-être dans l'économie.

\subsection{Règles budgétaires face aux chocs}

Les règles ont des implications différentes quant à la capacité de la politique budgétaire à répondre aux chocs. Au cours des années récentes plusieurs études économétriques ont tenté d'évaluer la performance des règles budgétaires. Ces études ont porté surtout sur les pays industrialisés et couvrent la période avant la crise financière mondiale de 2007-08. Une revue partielle de ces études est fournie par le Fonds monétaire international (2009); Elle suggère que les règles budgétaires nationales ont contribué, en général, à améliorer la performance en matière budgétaire. Certaines études ont trouvé que les gouvernements qui obéissent de manière stricte aux règles d'équilibre budgétaire connaissent des fluctuations des recettes et des dépenses au cours du cycle moins prononcé que les Gouvernements ayant des règles budgétaires moins rigoureuses. D'autres études ont montré que l'application stricte des règles budgétaires favorise l'épargne de précaution dans les périodes de haute conjoncture ; celle-ci peut ainsi être utilisée pour le financement des mesures budgétaires contra cycliques dans les phases de conjoncture défavorable. Cependant, les résultats sont en général moins probants particulièrement pour les pays de l'Union européenne, comme discuté tantôt en ce qui concerne les performances en matière de soutenabilité de la dette publique. Lié à ce résultat, les règles budgétaires ne semblent pas avoir un effet de crédibilité direct, en termes de primes de risque sur les marchés.

Nerlich et Reuter (2013) par exemple, dans une étude portant sur l'ensemble des pays membres de l'Union européenne et couvrant la période 1990-2012, ont trouvé que l'efficacité des règles peut effectivement être améliorée par la mise en place de comités budgétaires indépendants, 
notamment lorsque la nomination des membres de ces comités, et les ressources dont elles disposent, ne dépendent pas du gouvernement.

Maltritz et Wuste (2015) trouvent des résultats similaires pour le même groupe de pays au cours de la période 1991-2011, en utilisant des indices plus précis de la solidité des règles budgétaires basé sur les calculs de la Commission européenne (2013) évoqués tantôt et des comités indépendants. Leurs résultats, en conformité avec la littérature évoquée antérieurement, suggèrent que les règles budgétaires ont un impact positif sur les performances budgétaires. Par contre, les comités indépendants n'ont pas d'impact significatif direct. Toutefois, l'effet joint des règles et des comités est lui significatif, ce qui signifie essentiellement que l'efficacité des règles budgétaires est renforcée par la présence des comités budgétaires.

Reuter (2015), dans une étude sur 11 pays de l'Union européenne et couvrant la période 1994-2012, a trouvé que l'introduction des règles budgétaires a eu un effet contraignant significatif sur la politique budgétaire même si les objectifs ne sont atteints, en moyenne, que la moitié du temps dans l'année ou ils sont formulés.

Bergman et Hutchison (2015), dans une étude économétrique portant aussi bien sur les pays développés qu'en développement- sur un échantillon de 81 pays au cours de la période 1985- 2012. Les auteurs développent un indice de règles budgétaires, qui varie par pays et dans le temps basé sur 28 caractéristiques de ces règles, telles que contenues dans la base de données du Fond Monétaire International. Les résultats montrent que, conformément à plusieurs études antérieures, la pro- cyclicité des dépenses publiques est atténuée de manière significative par la présence des règles. De plus, lorsque les pays de l'échantillon sont désagrégés en fonction du degré d'efficacité de leur bureaucratie, ils trouvent que les règles budgétaires sont inefficaces dans la mesure où leurs effets sur la pro-cyclicité des dépenses sont négligeables. Sur le plan national, Tounsi et al. (2019) ont estimé via une modélisation structurelle en données de Panel à effet aléatoire, durant la période de 20002016, le niveau de pression fiscale optimale. Les résultats montrent que l'évolution annuelle du potentiel fiscal en moyenne, au Maroc durant la période d'étude est de $1,65 \%$, traduisant ainsi, un niveau de prélèvement effectif supérieur à la pression fiscale des pays de l'échantillon, et créant structurellement de ce fait, un espace budgétaire additionnel pour l'économie nationale.

\section{Effet des chocs des dépenses publiques sur l'activité économique au Maroc.}

4.1. Méthodologie empirique

La présentation du modèle choisi dans notre étude prend la forme d'un modèle VAR, jugé le plus efficace pour examiner les chocs budgétaires 
sur la croissance économique au Maroc. L'approche nommée dans ce travail va se baser sur l'estimation d'un modèle S-VAR permettant d'identifier les chocs structurels qui affectent le modèle de forme réduite grâce aux restrictions imposées dans les matrices de passage.

Dans cette étude, l'approche SVAR vise principalement à étudier les effets multiplicateurs différenciés des, chocs de dépenses d'investissement, des dépenses publiques générales ainsi que les dépenses publiques ordinaires (de consommation publique) sur l'activité économique au Maroc.

Cette approche standard est adoptée principalement pour analyser les effets multiplicateurs différenciés des chocs de dépenses publiques d'investissement et des dépenses publiques générales ainsi que les dépenses publiques ordinaires (de consommation publique) sur l'activité économique au Maroc.

Trois modélisations VAR ont été choisis pour expliquer la dynamique jointe du PIB réel $\left(P I B_{t}\right)$, ainsi que les dépenses publiques différenciées sont employées dans le but d'avoir une vision comparative dans les résultats : les dépenses publiques générales $\left(D P T_{t}\right)$, les dépenses publiques d'investissement $\left(D P I_{t}\right)$ et les dépenses publiques ordinaires $\left(D P O_{t}\right)$.

Pour un premier temps, l'approche méthodologique débute par la modélisation des trois modèles VAR avec un seul retard sur les variables à expliquer, afin de garantir une simplicité dans la formation de notre modèle.

Modèle VAR relatif aux dépenses publiques totales $\left(D P T_{t}\right)$ :

$$
\left\{\begin{array}{l}
\mathrm{PIB}_{\mathrm{t}}+\beta_{12} \mathrm{DPT}_{\mathrm{t}}=\delta_{10}+\delta_{11} \mathrm{PIB}_{\mathrm{t}-1}+\delta_{12} \mathrm{DPT}_{\mathrm{t}-1}+\varepsilon_{\mathrm{PIB}_{\mathrm{t}}} \\
\beta_{21} \mathrm{PIB}_{\mathrm{t}}+\mathrm{DPT}_{\mathrm{t}}=\delta_{20}+\delta_{21} \mathrm{PIB}_{\mathrm{t}-1}+\delta_{22} \mathrm{DPT}_{\mathrm{t}-1}+\varepsilon_{\mathrm{DPT}_{\mathrm{t}}}
\end{array}\right.
$$

Modèle VAR relatif aux dépenses publique d'investissement $\left(D P I_{t}\right)$ :

$$
\left\{\begin{array}{l}
\mathrm{PIB}_{\mathrm{t}}+\beta_{12} \mathrm{DPI}_{\mathrm{t}}=\delta_{10}+\delta_{11} \mathrm{PIB}_{\mathrm{t}-1}+\delta_{12} \mathrm{DPI}_{\mathrm{t}-1}+\varepsilon_{\mathrm{PIB}_{\mathrm{t}}} \\
\beta_{21} \mathrm{PIB}_{\mathrm{t}}+\mathrm{DPI}_{\mathrm{t}}=\delta_{20}+\delta_{21} \mathrm{PIB}_{\mathrm{t}-1}+\delta_{22} \mathrm{DPI}_{\mathrm{t}-1}+\varepsilon_{\mathrm{DPI}_{\mathrm{t}}}
\end{array}\right.
$$

Modèle VAR relatif aux dépenses publique ordinaire $\left(D P O_{t}\right)$ :

$$
\left\{\begin{array}{l}
\mathrm{PIB}_{\mathrm{t}}+\beta_{12} \mathrm{DPO}_{\mathrm{t}}=\delta_{10}+\delta_{11} \mathrm{PIB}_{\mathrm{t}-1}+\delta_{12} \mathrm{DPO}_{\mathrm{t}-1}+\varepsilon_{\mathrm{PIB}_{\mathrm{t}}} \\
\beta_{21} \mathrm{PIB}_{\mathrm{t}}+\mathrm{DPO}_{\mathrm{t}}=\delta_{20}+\delta_{21} \mathrm{PIB}_{\mathrm{t}-1}+\delta_{22} \mathrm{DPO}_{\mathrm{t}-1}+\varepsilon_{\mathrm{DPO}_{\mathrm{t}}}
\end{array}\right.
$$

Après l'exposition des trois modèles VAR, il est nécessaire de les convertir en forme matricielle pour construire un modèle réduit, donc les VAR structurelles peuvent s'écrire suivant une forme unique, d'où la présentation suivante prend une seule forme matricielle correspondante au modèle VAR relatif aux dépenses publiques totales, alors l'écriture matricielle se présente comme suit : 


$$
\left[\begin{array}{cc}
1 & \beta_{12} \\
\beta_{21} & 1
\end{array}\right] \cdot\left[\begin{array}{c}
\mathrm{PIB}_{\mathrm{t}} \\
\mathrm{DPT}_{\mathrm{t}}
\end{array}\right]=\left[\begin{array}{c}
\delta_{10} \\
\delta_{20}
\end{array}\right]+\left[\begin{array}{cc}
\delta_{11} & \delta_{12} \\
\delta_{21} & \delta_{22}
\end{array}\right] \cdot\left[\begin{array}{c}
\mathrm{PIB}_{\mathrm{t}-1} \\
\mathrm{DPT}_{\mathrm{t}-1}
\end{array}\right]+\left[\begin{array}{c}
\varepsilon_{\mathrm{PIB}_{\mathrm{t}}} \\
\varepsilon_{\mathrm{DPT}_{\mathrm{t}}}
\end{array}\right]
$$

Avec : $\Omega=\left[\begin{array}{cc}\sigma_{\mathrm{PIB}}^{2} & 0 \\ 0 & \sigma_{\mathrm{DPT}}^{2}\end{array}\right]$ et $\varepsilon_{\mathrm{t}}=\left[\begin{array}{c}\varepsilon_{\mathrm{PIB}_{\mathrm{t}}} \\ \varepsilon_{\mathrm{DPT}_{\mathrm{t}}}\end{array}\right]$

Sont respectivement la matrice variance covariance des termes d'erreur $\varepsilon_{t}$, supposées être diagonales par hypothèses, et le vecteur $\varepsilon_{t}$ est un vecteur de chocs structurels indépendants ayant des effets contemporains sur les variables du modèle. Ces chocs sont indépendants et sans corrélations temporelles à toutes les horizons. Le VAR structurel peut s'écrire d'une manière plus compacte suivant la forme suivante :

$$
\text { B. } Z_{\mathrm{t}}=\tau_{0}+\mathrm{M}_{1} \cdot \mathrm{Z}_{\mathrm{t}-1}+\varepsilon_{\mathrm{t}}
$$

Avec :

$$
\begin{gathered}
B=\left[\begin{array}{cc}
1 & \beta_{12} \\
\beta_{21} & 1
\end{array}\right], Z=\left[\begin{array}{l}
P I B_{t} \\
D P T_{t}
\end{array}\right], \tau_{0}=\left[\begin{array}{l}
\delta_{10} \\
\delta_{20}
\end{array}\right], M_{1}=\left[\begin{array}{ll}
\delta_{11} & \delta_{12} \\
\delta_{21} & \delta_{22}
\end{array}\right], Z_{t-1}=\left[\begin{array}{l}
P I B_{t-1} \\
D P T_{t-1}
\end{array}\right] \\
\text { Et } \quad \varepsilon_{t}=\left[\begin{array}{l}
\varepsilon_{P I B_{t}} \\
\varepsilon_{D P T_{t}}
\end{array}\right]
\end{gathered}
$$

Les paramètres du VAR structurel ne peuvent pas être estimés directement par la méthode des MCO en raison de la corrélation induite par le modèle entre les variables explicatives et les termes d'erreur (c'est ce qui explique pourquoi l'estimation d'un modèle VAR structurel par MCO mène à des estimateurs biaisés), pourtant cette difficulté peut être contournée en multipliant les coefficients de cette égalité par la matrice inverse $B^{-1}$,tout en transformant le VAR structurel en VAR standard.

Le modèle VAR standard s'écrit comme suit :

$$
\begin{gathered}
\mathrm{Z}_{\mathrm{t}}=\mathrm{B}^{-1} \tau_{0}+\mathrm{B}^{-1} \tau_{1} \cdot \mathrm{Z}_{\mathrm{t}-1}+\mathrm{B}^{-1} \varepsilon_{\mathrm{t}} \\
\mathrm{Z}=\left[\begin{array}{l}
\mathrm{PIB}_{\mathrm{t}} \\
\mathrm{DPT}_{\mathrm{t}}
\end{array}\right], \quad \mathrm{B}^{-1} \tau_{0}=\mathrm{M}_{0}, \quad \mathrm{~B}^{-1} \tau_{1}=\mathrm{M}_{1}, \mathrm{Z}_{\mathrm{t}-1}=\left[\begin{array}{l}
\mathrm{PIB} \mathrm{B}_{\mathrm{t}-1} \\
\mathrm{DPT} \mathrm{T}_{\mathrm{t}-1}
\end{array}\right] \quad \text { Et } \quad \mathrm{B}^{-1} \varepsilon_{\mathrm{t}}=\mathrm{U}_{\mathrm{t}}
\end{gathered}
$$

Alors,

$$
\mathrm{Z}_{\mathrm{t}}=\mathrm{M}_{0}+\mathrm{M}_{1} \cdot \mathrm{Z}_{\mathrm{t}-1}+\mathrm{U}_{\mathrm{t}}
$$

Tout en identifiant que $\Sigma=\left[\begin{array}{ll}\delta_{11} & \delta_{12} \\ \delta_{21} & \delta_{22}\end{array}\right]$ comme la matrice de variance-covariance du vecteur $U_{t}$ des erreurs du VAR standard. Le modèle VAR standard peut s'écrire également comme un système d'équations de la forme suivante :

$$
\left\{\begin{array}{l}
\mathrm{PIB}_{\mathrm{t}}=\alpha_{10}+\alpha_{11} \mathrm{PIB}_{\mathrm{t}-1}+\alpha_{12} \mathrm{DPT}_{\mathrm{t}-1}+\mathrm{U}_{1 \mathrm{t}} \\
\mathrm{DPT}_{\mathrm{t}}=\alpha_{20}+\alpha_{21} \mathrm{PIB}_{\mathrm{t}-1}+\alpha_{22} \mathrm{DPT}_{\mathrm{t}-1}+\mathrm{U}_{2 \mathrm{t}}
\end{array}\right.
$$

Dans ce modèle basé sur l'approche utilisée par Sims (1980), et repris par Enders (2010), la matrice $M_{1}$ est soumise aux conditions initiales de 
stabilité et de stationnarité des variables macroéconomiques et budgétaires du modèle. Il est important de noter que le vecteur Ut du modèle dans la forme réduite n'est pas le vecteur de chocs structurels fondamentaux du modèle VAR structurel. Le vecteur $U_{t}$ est plutôt un vecteur de chocs qui sont à la fois de moyenne nulle, de variance constante et individuellement sans corrélation sérielle. Cependant, la covariance des chocs du VAR standard est non nulle aussi longtemps que les coefficients $\beta_{12}$ et $\beta_{21}$ du VAR structurel sont différents de zéro, c'est-à-dire si les variables $D P T_{t}$ et $P I B_{t}$ sont déterminées simultanément.

Une telle approche a été expérimentée par Perotti et al (2007) quant à l'identification des chocs budgétaires. Partant de cette approche standard dans la littérature macro- économétrique sur les VAR structurels, la relation entre les vecteurs résiduels et ceux des chocs structurels des variables budgétaires s'écrit sous la forme réduite de l'équation ci-après :

$$
\text { R. } U_{t}=K \cdot V_{t}
$$

Dans cette forme réduite le $U_{t}$ et $V_{t}$ désignent respectivement les vecteurs des résidus du VAR standard et des chocs structurels des variables du modèle, tandis que $\mathrm{R}$ et $\mathrm{K}$ sont les matrices de covariance de distribution des résidus et de chocs.

\subsection{Identification des restrictions}

Dans une forme plus détaillée le VAR structurel peut s'écrire sous forme matricielle :

$$
\begin{gathered}
\left(\begin{array}{cc}
1 & -\alpha_{12} \\
-\alpha_{21} & 1
\end{array}\right) \cdot\left(\begin{array}{c}
\mathrm{U}_{\mathrm{PIB}_{\mathrm{t}}} \\
\mathrm{U}_{\mathrm{DPT}_{\mathrm{t}}}
\end{array}\right)=\left(\begin{array}{cc}
1 & \beta_{12} \\
\beta_{21} & 1
\end{array}\right) \cdot\left(\begin{array}{c}
\varepsilon_{\mathrm{PIB}_{\mathrm{t}}} \\
\varepsilon_{\mathrm{DPT}_{\mathrm{t}}}
\end{array}\right) \\
\mathrm{A}=\left(\begin{array}{cc}
1 & -\alpha_{12} \\
-\alpha_{21} & 1
\end{array}\right), \quad \mathrm{B}=\left(\begin{array}{cc}
1 & \beta_{12} \\
\beta_{21} & 1
\end{array}\right)
\end{gathered}
$$

L'identification du modèle VAR structurel considéré revient à imposer une restriction sur le modèle VAR structurel qui va être illustré dans la matrice des restrictions (A). La restriction relative aux modèles VAR correspond à ce que les variables budgétaires aient des effets économiques contemporains sur les variables macroéconomiques qui, à leur tour, affectent les variables budgétaires avec une période de retard. Autrement dit, les variables macroéconomiques n'ont pas d'effets contemporains sur les variables budgétaires, Ainsi, sous l'hypothèse que l'effet contemporain du revenu (PIB) réel sur les dépenses publiques totales est nul $\left(\alpha_{21}=0\right)$.

Sur la base de la théorie économique et de certaines études empiriques notamment celles de Blanchard et Perotti (1999), l'élasticité des dépenses publiques totales par rapport aux PIB est supposée égale a $\left(\alpha_{21}=\right.$ 0,9 ), ladite élasticité est extraite d'une étude du ministère de l'économie et des finances. D'après cette étude on trouve que l'élasticité était d'environ 0,9 
sur la période 1990-1995. Nous avons effectué des estimations avec une élasticité de 0,9 de 1 et de 1,1. Les résultats montrent que le meilleur choix est de 0,9 . De ce fait, le nouveau modèle s'écrit comme suit :

$$
\left(\begin{array}{cc}
1 & -0,9 \\
0 & 1
\end{array}\right) \cdot\left(\begin{array}{c}
\mathrm{U}_{\mathrm{PIB}_{\mathrm{t}}} \\
\mathrm{U}_{\mathrm{DPT}_{\mathrm{t}}}
\end{array}\right)=\left(\begin{array}{cc}
1 & \beta_{12} \\
\beta_{21} & 1
\end{array}\right) \cdot\left(\begin{array}{l}
\varepsilon_{\mathrm{PIB}_{\mathrm{t}}} \\
\varepsilon_{\mathrm{DPT}_{\mathrm{t}}}
\end{array}\right)
$$

\subsection{Données}

Sur la base des données annuelles du PIB réel, des dépenses publiques ordinaires, des dépenses d'investissement et des dépenses totales sur la période allant de 1970 à 2019, 1'approche SVAR a été adoptée pour examiner les effets des chocs budgétaires sur l'activité économique au Maroc.

Tableau 1. Description et source des variables

\begin{tabular}{llll}
\hline Variable & Description & Période & Source \\
\hline PIB & PIB réel & $1970-2019$ & $\begin{array}{l}\text { Ministère d'économie } \\
\text { et des finances - DEPF }\end{array}$ \\
\hline DO & Dépenses publiques ordinaires & $1970-2019$ & $\begin{array}{l}\text { Ministère d'économie } \\
\text { et des finances - DEPF }\end{array}$ \\
\hline INV & $\begin{array}{l}\text { Dépenses } \\
\text { d'investissement }\end{array}$ publiques & $1970-2019$ & $\begin{array}{l}\text { Ministère d'économie } \\
\text { et des finances - DEPF }\end{array}$ \\
\hline DPT & Dépenses publiques totales & $1970-2019$ & $\begin{array}{l}\text { Ministère d'économie } \\
\text { et des finances - DEPF }\end{array}$ \\
\hline
\end{tabular}

Les résultats des tests de racine unitaire (ADF) indiquent que les variables du modèle sont non stationnaires en niveau (Annexe 1). De ce faite, la transformation des variables en différence première a conduit à la stationnarité de ces dernières, donc elles sont intégrées du même ordre.

\subsection{Résultats et interprétations}

Les résultats montrent que les chocs de dépenses publiques d'investissement ont un effet persistent sur le PIB réel. En effet les chocs de dépenses ordinaires ont un effet immédiat plus important que les chocs de dépenses publiques d'investissement et les dépenses publiques totales. En gros, les effets multiplicateurs d'un choc positif de dépenses ordinaires sur le PIB réel sont plus importants à la période actuelle que ceux d'un choc de dépenses publiques d'investissement. Ce résultat rejoint la critique de Lucas (1976) à propos de la théorie des cycles macroéconomiques selon laquelle, la sensibilité du PIB réel aux chocs de dépenses publiques varie avec la nature de ces chocs. 
Figure 1. Fonctions de réponses des modèles SVAR
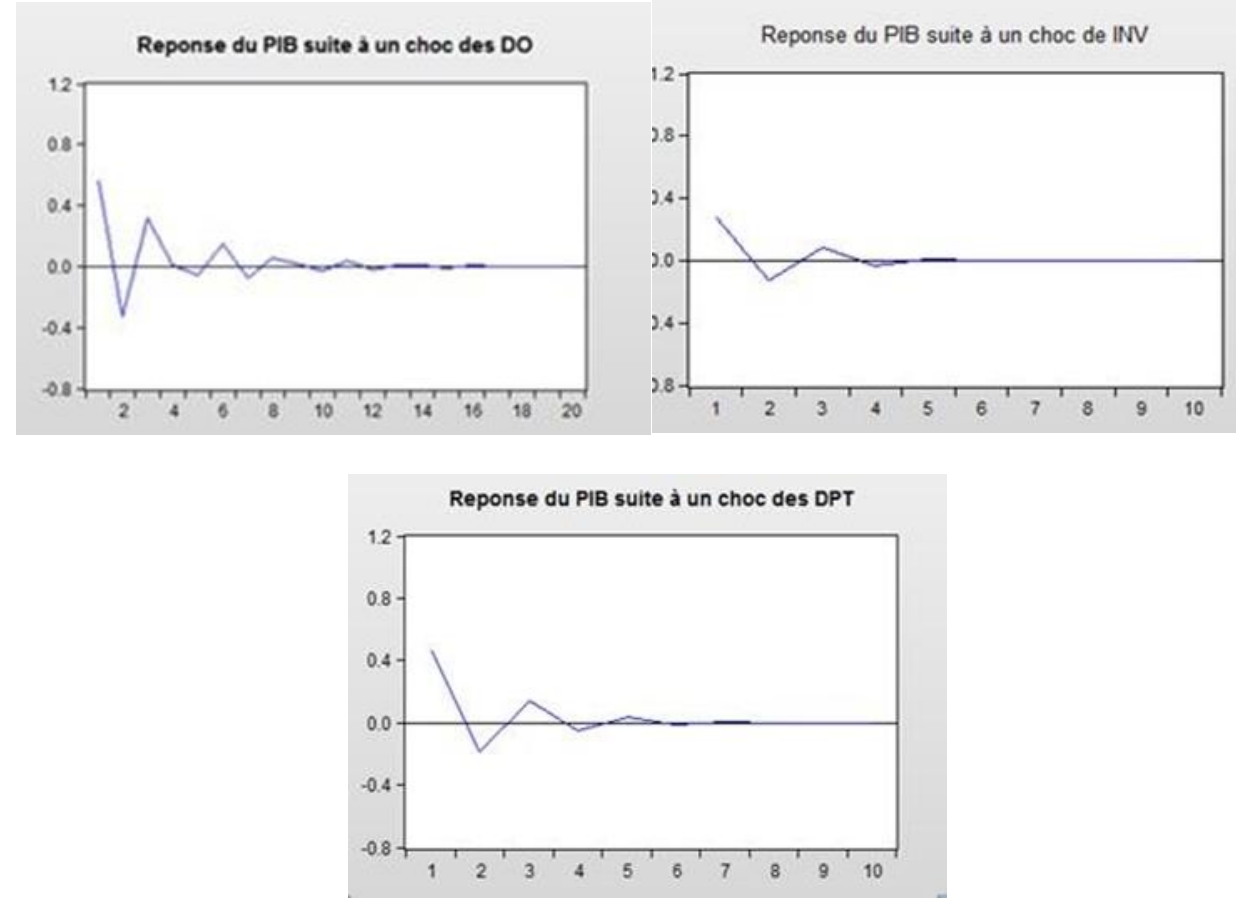

Source : Par nos propres soins sous Eviews

Les résultats d'estimation des effets multiplicateurs suite aux chocs budgétaires sur la variable macroéconomique montrent que les effets multiplicateurs des chocs des dépenses d'investissement sur le PIB réel différent des chocs des dépenses ordinaires et aussi des dépenses publiques totales par comparaison de la fonction de réponse du PIB réel aux chocs budgétaires à court terme.

Les effets multiplicateurs d'un choc positif des trois variables budgétaires sur le PIB réel sont tous positifs à la période du choc.

Une augmentation des dépenses d'investissement de $1 \%$ s'accompagne d'une augmentation de $0.28 \%$ du PIB réel Marocain. Ce résultat confirme l'intuition économique que les chocs de dépenses d'investissement stimulent la conjoncture économique.

Ainsi que les chocs positifs sur les dépenses ordinaires et sur les dépenses totales ont des effets contemporains positifs de 0.43 et 0.56 respectivement.

En effet, les effets contemporains des chocs de dépenses ordinaires sont plus importants que ceux des dépenses d'investissement et des dépenses totales.

Le premier test à effectuer est celui vérifiant la stationnarité : l'application des différents tests de stationnarité pour les trois séries 
considérées mène aux résultats regroupés dans le tableau en annexes. (Annexe 1).

Les résultats des tests de racine unitaire (ADF) montrent que les variables du modèle sont stationnaires en différence première, donc elles sont intégrées du même ordre.

Pour le choix du retard optimal, nous avons utilisé les résultats présentés dans le tableau (Annexe 2) qui conduisent à une spécification à un seul retard.

Les lignes du Tableau (annexe 3) présentent les résultats du test d'absence de corrélation temporelle des résidus du VAR. Dans les trois cas, à partir d'une formulation avec 10 retards, l'hypothèse nulle d'absence de corrélation temporelle ne peut être rejetée au seuil habituel de 5\%. Ceci dit, on peut donc conclure que les résultats du test d'auto corrélation des résidus du VAR standard n'indiquent pas la présence d'erreurs de spécifications dans la structure de retards des différents modèles.

\section{Conclusion}

L'objectif du présent article est d'analyser l'impact des chocs budgétaires sur l'activité économique au Maroc. Pour ce faire, une première partie s'est attelée à présenter d'une façon ordonnée les débats théoriques autour de la question de la performance des règles de la politique budgétaire. Ainsi, il en ressort que cette performance s'illustre dans le fait qu'elles évitent les déséquilibres structurels persistant des finances publiques, limitent le caractère pro cyclique des politiques publiques, et maintient les ratios de dette publique à des niveaux soutenables. Par la suite, quelques travaux empiriques dont les préoccupations sont semblables à la présente étude ont été exposés.

En résumé, l'étude effectuée s'est focalisé sur l'analyse des multiplicateurs budgétaires selon différents types de choc, à savoir le choc sur les dépenses d'investissement, dépenses ordinaires et sur les dépenses totales, afin de visualiser la réaction de l'activité économique suite à ces chocs.

L'approche utilisée dans ce travail est basée sur une modélisation en SVAR permettant d'étudier les effets multiplicateurs différenciés des, chocs de dépenses d'investissement, des dépenses publiques générales ainsi que les dépenses publiques ordinaires (de consommation publique) sur l'activité économique au Maroc. Les résultats des trois modèles SVAR proposés dans le cadre de cette étude ont tous conclus à un impact positif. En effet, l'effet multiplicateur le plus important est celui relatif aux chocs de dépenses ordinaires par rapport aux chocs de dépenses d'investissement et des dépenses totales. Ces résultats rejoignent la critique de Lucas à propos de la théorie des cycles macroéconomiques selon laquelle, la sensibilité du PIB réel aux chocs de dépenses publiques varie avec la nature de ces chocs. 
Dans ce cadre, la situation des finances publiques du Maroc durant la période étudiée (1970-2019), montre une volatilité du solde ordinaire, chose qui a empêché de soustraire une politique budgétaire ayant le caractère d'une règle. Poursuivant une politique d'austérité et d'assainissement de la situation financière de l'État, ce programme est de nature essentiellement récessionniste (baisse des dépenses). Certes, il a permis de ramener le taux de croissance des dépenses courantes, et de baisser le montant des dépenses d'investissement de $40 \%$ en 1978 , mais celles-ci sont restées à un niveau élevé à cause de la rigidité structurelle caractérisant certains investissements. En effet, la dette publique par rapport au PIB a plus que doublé entre 1974 et 1981 passant ainsi de 22,4\% à 53,38\%. Malgré l'assainissement budgétaire prôné par le Plan de stabilisation 1978-1980, les déficits budgétaires ont continué à persister, surtout en 1981 représentant 14\% du PIB. La baisse des dépenses d'équipement et les divers aménagements fiscaux n'ont guère contribué à améliorer la situation financière de l'État. Celle-ci s'est même dégradée à partir de 1980 avec l'apparition des soldes budgétaires ordinaires négatifs. Le Programme n'a pas pu être maintenu au-delà de 1979 à cause de la détérioration des indicateurs économiques et financiers. Au début des années 1980, le Maroc s'est trouvé confronté à des pressions financières aiguës et à une détérioration prononcée des équilibres internes et externes. Ces déséquilibres trouvent leur origine dans les faiblesses structurelles de l'économie nationale liées, notamment, à la forte dépendance de la production agricole des aléas climatiques, à la faible dynamique industrielle, à la vulnérabilité des exportations à l'égard des cours internationaux, en particulier pour les phosphates, auxquelles s'est ajoutée une conjoncture.

Afin d'étudier la problématique en question tout en prenant compte l'ensemble des faits stylisés de l'économie marocaine à savoir les évolutions des finances publiques et les différents comportements des agents économiques, la littérature révèle l'utilisation d'un modèle en panel VAR pour une analyse en benchmark. Une autre piste peut être proposée, qui propose le passage à un modèle $\mathrm{DSGE}^{3}$ afin de capter le comportement de l'ensemble des agents économiques et de voir la dynamique des effets des dépenses publiques dans le temps.

\section{References :}

1. Alesina, A., \& Perotti, R. (1994). The political economy of growth: a critical survey of the recent literature. The World Bank Economic Review, 8(3), 351-371.

2. Barro, R. J. (1981). Output effects of government purchases. Journal of political Economy, 89(6), 1086-1121.

${ }^{3}$ Modèle d'équilibre général dynamique stochastique 
3. Baxter, M., \& King, R. G. (1993). Fiscal policy in general equilibrium. The American Economic Review, 315-334.

4. Bergman, U. M., \& Hutchison, M. (2015). Economic stabilization in the post-crisis world: Are fiscal rules the answer?. Journal of International Money and Finance, 52, 82-101.

5. Bergman, U. M., \& Hutchison, M. (2015). Economic stabilization in the post-crisis world: Are fiscal rules the answer? Journal of International Money and Finance, 52, 82-101.

6. Blanchard, O., \& Perotti, R. (1999). An empirical characterization of the dynamic effects of changes in government spending and taxes on output NBER working paper, 7269.

7. Blanchard, O., \& Perotti, R. (2002). An empirical characterization of the dynamic effects of changes in government spending and taxes on output. the Quarterly Journal of economics, 117(4), 1329-1368.

8. Cogan, J. F., Cwik, T., Taylor, J. B., \& Wieland, V. (2010). New Keynesian versus old Keynesian government spending multipliers. Journal of Economic dynamics and control, 34(3), 281295.

9. Corsetti, G., \& Roubini, N. (1996). Optimal government spending and taxation in endgenous growth models (No. w5851). National bureau of economic research.

10. Drazen, A. (2004). Political economy in macro-economics. Orient Blackswan.

11. Enders, C. K. (2010). Applied missing data analysis. Guilford press.

12. Kydland, F. E., \& Prescott, E. C. (1977). Rules rather than discretion: The inconsistency of optimal plans. Journal of political economy, 85(3), 473-491.

13. Maltritz, D., \& Wüste, S. (2015). Determinants of budget deficits in Europe: The role and relations of fiscal rules, fiscal councils, creative accounting and the Euro. Economic Modelling, 48, 222-236.

14. Mathieu, C., \& Sterdyniak, H. (2009). Quelles stratégies budgétaires de sortie de crise ?.

15. Nerlich, C., \& Reuter, W. H. (2013). The design of national fiscal rules and their budgetary impact. ECB working paper series 1588 .

16. Perotti, R., Reis, R., \& Ramey, V. (2007). In search of the transmission mechanism of fiscal policy [with comments and discussion]. NBER macroeconomics Annual, 22, 169-249.

17. Reuter, W. H. (2015). National numerical fiscal rules: Not complied with, but still effective?. European Journal of Political Economy, 39, 67-81.

18. Sims, C. A. (1980). Macroeconomics and reality. Econometrica: journal of the Econometric Society, 1-48. 
19. Tounsi, S., Fadllalah, A., \& Wahbi, A. (2019). De L'effort Au Potentiel Fiscal Au Maroc: Evaluation Empirique. European Scientific Journal, ESJ, 15(34), 342.

20. Woodford, M. (2010). Financial intermediation and macroeconomic analysis. Journal of Economic Perspectives, 24(4), 21-44.

\section{Annexes}

\section{Test de stationnarité}

\begin{tabular}{|c|c|c|c|}
\hline Variables & En niveau & En première Différence & Ordre d'intégration \\
\hline PIB & $\mathbf{- 2 , 5 1}$ & $\mathbf{- 5 , 0 3}$ & $\mathbf{I}(\mathbf{1})$ \\
\hline DPI & $\mathbf{- 2 , 5 8}$ & $\mathbf{- 5 , 3 6}$ & $\mathbf{I}(\mathbf{1})$ \\
\hline DPT & $\mathbf{- 2 , 3 5}$ & $\mathbf{- 6 , 0 5}$ & $\mathbf{I}(\mathbf{1})$ \\
\hline DPO & $\mathbf{- 3 , 1 8}$ & $\mathbf{- 7 , 4 5}$ & $\mathbf{I}(\mathbf{1})$ \\
\hline
\end{tabular}

\section{Choix du retard optimal}

\section{VAR Lag Order Selection Criteria}

Endogenous variables: DLPIB DLDPT

Exogenous variables: $\mathrm{C}$

Date: 06/04/16 Time: 00:35

Sample: 19702012

Included observations: 37

\begin{tabular}{ccccccc}
\hline \hline Lag & LogL & LR & FPE & AIC & SC & HQ \\
\hline \hline 0 & 106.0560 & NA & $1.24 \mathrm{e}-05$ & -5.624646 & -5.537570 & -5.593948 \\
1 & 114.7809 & $16.03507^{\star}$ & $9.59 \mathrm{e}-06^{\star}$ & $-5.880050^{\star}$ & $-5.618820^{\star}$ & $-5.787954^{\star}$ \\
2 & 116.5244 & 3.015704 & $1.09 \mathrm{e}-05$ & -5.758074 & -5.322691 & -5.604581 \\
3 & 118.0391 & 2.456267 & $1.25 \mathrm{e}-05$ & -5.623734 & -5.014197 & -5.408844 \\
4 & 121.2832 & 4.910017 & $1.32 \mathrm{e}-05$ & -5.582875 & -4.799185 & -5.306588 \\
5 & 124.6531 & 4.736063 & $1.38 \mathrm{e}-05$ & -5.548815 & -4.590972 & -5.211131 \\
\hline \hline
\end{tabular}

* indicates lag order selected by the criterion

LR: sequential modified LR test statistic (each test at $5 \%$ level)

FPE: Final prediction error

AIC: Akaike information criterion

SC: Schwarz information criterion

$\mathrm{HQ}$ : Hannan-Quinn information criterion 


\section{Test d'absence de corrélation temporelle des résidus des modèles}

\begin{tabular}{|c|c|c|}
\hline Lags & LM-Stat & Prob \\
\hline 1 & 2.558369 & 0.6342 \\
\hline 2 & 3.233638 & 0.5195 \\
\hline 3 & 2.951936 & 0.5659 \\
\hline 4 & 4.664300 & 0.3235 \\
\hline 5 & 1.201579 & 0.8778 \\
\hline 6 & 0.573502 & 0.9660 \\
\hline 7 & 9.721588 & 0.0454 \\
\hline 8 & 3.441674 & 0.4868 \\
\hline 9 & 1.857587 & 0.7619 \\
\hline 10 & 0.769009 & 0.9426 \\
\hline
\end{tabular}

Probs from chi-square with $4 \mathrm{df}$.

4. Effets contemporains des variables budgétaires sur le PIB Marocain

\begin{tabular}{|c|c|c|c|c|c|c|c|c|}
\hline \multicolumn{3}{|c|}{ Dépenses d'investissement } & \multicolumn{3}{|c|}{ Dépenses ordinaires } & \multicolumn{3}{|c|}{ Dépenses totales } \\
\hline \multicolumn{3}{|c|}{ Response of DLPIB: } & \multicolumn{3}{|c|}{ Response of DLPIB: } & \multicolumn{3}{|c|}{ Response of DLPIB: } \\
\hline Period & Shock1 & Shock2 & Perioc & Shock1 & Shock2 & Period & Shock1 & Shock2 \\
\hline 1 & 0.279332 & 1.000000 & 1 & 0.562369 & 1.000000 & 1 & 0.465207 & 1.000000 \\
\hline 2 & -0.128492 & -0.517242 & $\begin{array}{l}2 \\
3\end{array}$ & $\begin{array}{r}-0.331845 \\
0.316534\end{array}$ & $\begin{array}{r}-0.596531 \\
0.542601\end{array}$ & 2 & -0.190765 & -0.445349 \\
\hline 3 & 0.079695 & 0.305373 & 4 & 0.013039 & 0.012683 & 3 & 0.137253 & 0.297221 \\
\hline 4 & -0.034941 & -0.132801 & 5 & -0.059384 & -0.102880 & 4 & -0.055769 & -0.124986 \\
\hline 5 & 0.013247 & 0.050616 & 6 & 0.152593 & 0.261514 & 5 & 0.032837 & 0.070743 \\
\hline 6 & -0.004837 & -0.018391 & $\begin{array}{l}7 \\
8\end{array}$ & $\begin{array}{r}-0.078261 \\
0.051989\end{array}$ & $\begin{array}{r}-0.139188 \\
0.090732\end{array}$ & 6 & -0.013977 & -0.030806 \\
\hline 7 & 0.001618 & 0.006132 & 9 & 0.016203 & 0.025869 & 7 & 0.007607 & 0.016385 \\
\hline 8 & -0.000494 & -0.001869 & 10 & -0.024680 & -0.042993 & 8 & -0.003367 & -0.007368 \\
\hline 9 & 0.000136 & 0.000513 & 11 & 0.035918 & 0.061996 & 9 & 0.001756 & 0.003787 \\
\hline 10 & $-3.14 E-05$ & -0.000117 & $\begin{array}{l}12 \\
13\end{array}$ & $\begin{array}{r}-0.016248 \\
0.006955\end{array}$ & $\begin{array}{r}-0.028978 \\
0.012274\end{array}$ & 10 & -0.000799 & -0.001743 \\
\hline & & & 14 & 0.006803 & 0.011378 & & & \\
\hline \multicolumn{3}{|c|}{ Factorization: Structural } & 15 & -0.007670 & -0.013373 & \multicolumn{3}{|c|}{ Factorization: Structural } \\
\hline & & & \multicolumn{3}{|c|}{ Factorization: Structural } & & & \\
\hline
\end{tabular}


February 2021 edition Vol.17, No.5

\section{Test de stabilité des modèles}

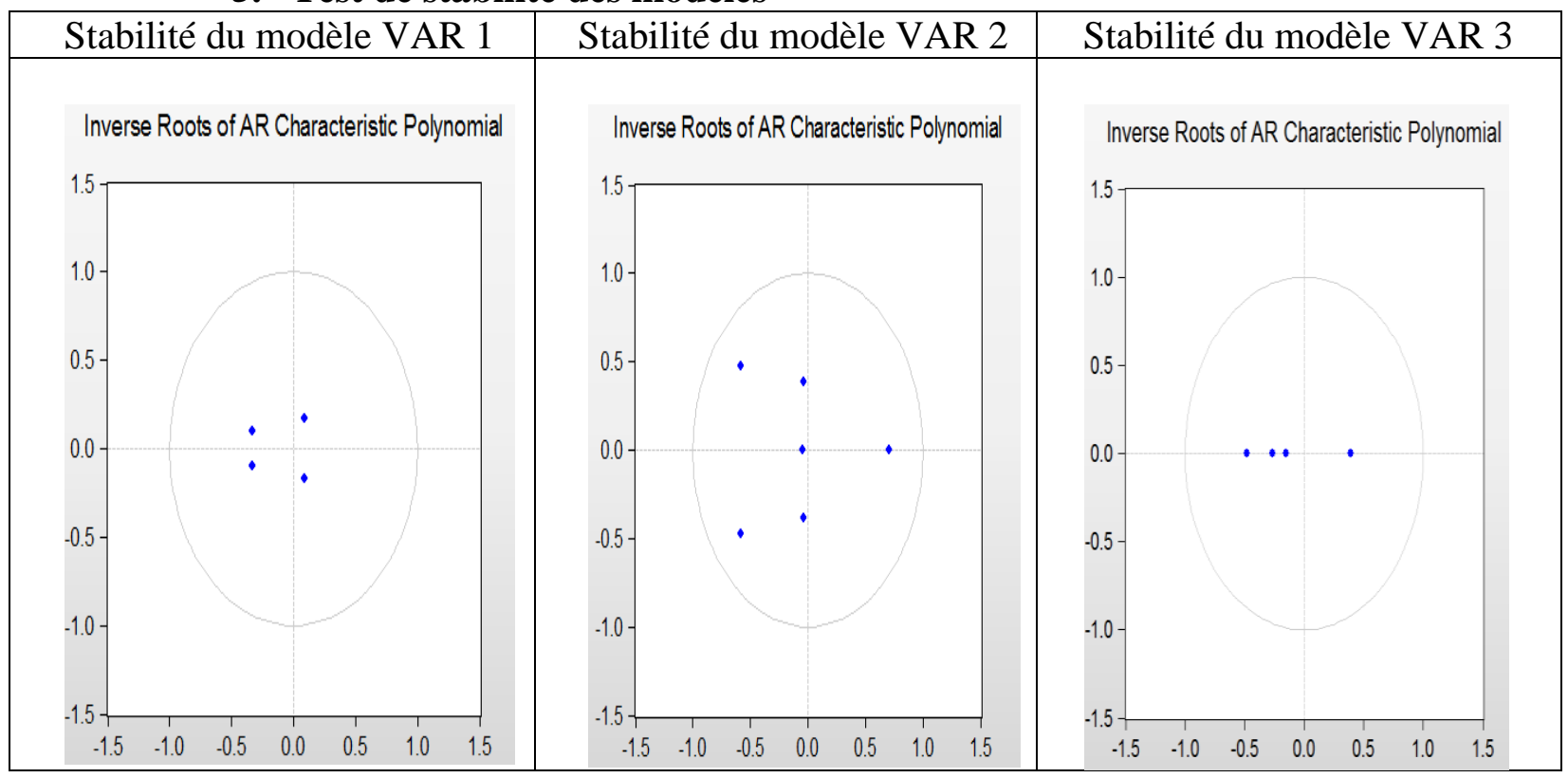

